\title{
Optimizing return-on-effort for coral nursery and outplanting practices to aid restoration of the Great Barrier Reef
}

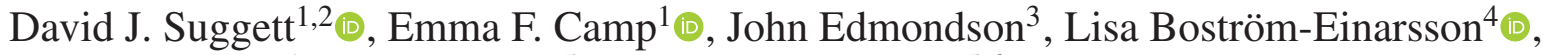 \\ Vanessa Ramler ${ }^{1}$, Kathryn Lohr $^{5}$, Joshua T. Patterson ${ }^{5,6}$
}

\begin{abstract}
Coral nursery and outplanting practices have grown in popularity worldwide for targeted restoration of degraded "high value" reef sites, and recovery of threatened taxa. Success of these practices is commonly gauged from coral propagule growth and survival, which fundamentally determines the return-on-effort (RRE) critical to the cost-effectiveness and viability of restoration programs. In many cases, RRE has been optimized from past successes and failures, which therefore presents a major challenge for locations such as the Great Barrier Reef (GBR) where no local history of restoration exists to guide best practice. In establishing the first multi-taxa coral nursery on the GBR (Opal Reef, February 2018), we constructed a novel scoring criterion from concurrent measurements of growth and survivorship to guide our relative RRE, including nursery propagule numbers (stock density). We initially retrieved RRE scores from a database of global restoration efforts to date ( $n=246 ; 52$ studies) to evaluate whether and how success commonly varied among coral taxa. We then retrieved RRE scores for Opal Reef using initial growth and survivorship data for six key coral taxa, to demonstrate that RRE scores were high for all taxa predominantly via high survivorship over winter. Repeated RRE scoring in summer is therefore needed to capture the full dynamic range of success where seasonal factors regulating growth versus survivorship differ. We discuss how $R R E$ scoring can be easily adopted across restoration practices globally to standardize and benchmark success, but also as a tool to aid decision-making in optimizing future propagation (and outplanting) efforts.
\end{abstract}

Key words: coral nursery, Great Barrier Reef, growth, reef restoration, return-on-effort, survivorship

\section{Implications for Practice}

- Reef restoration success is commonly gauged as yields of coral biomass achieved from growth and survival of nursery-reared and outplanted coral.

- Concurrent measurements of growth and survivorship enable relative return-on-effort (RRE) (i.e. relative to cost) to be scored, and thus a standardized means to compare success across practice, coral species, and growth environment.

- Retrieving RRE scoring from past "global" restoration efforts demonstrates how success inherently varies among coral taxa, and hence informs optimized propagule numbers.

- Applying RRE scoring to the first multi-taxa coral nursery on the Great Barrier Reef reveals that repeat RRE scoring is needed to capture environmental variability.

- Improved standardization of growth and survivorship measurements will enable RRE scoring to be embedded in global reef restoration success benchmarking.

\section{Introduction}

Accelerating coral reef degradation worldwide is driving management portfolios to increasingly adopt interventions that enhance coral stress resilience or fast-track reef recovery (Anthony et al. 2017). In situ coral propagation practices that boost coral abundance are becoming particularly popular to restore degraded "high value" reef sites that support local subsistence and reef-derived economies (Bayraktarov et al. 2016; Hein et al. 2017). Expansion of coral nursery and outplanting programs throughout the Caribbean, Indo-Pacific, and Red Sea over the past two decades has resulted in a wealth of knowledge for increasing coral biomass yields through propagation of fragmented adult colonies or of larvae (e.g. Clark \& Edwards 1995; Rinkevich 2000; Omori et al. 2007; Boström-Einarsson

Author contributions: DJS, EFC, JE conceived and designed the research; DJS, EFC, JE collected field data; DJS, LBE, KL, JP compiled literature metadata; DJS, VR analyzed the data; DJS led the writing, with all authors contributing through subsequent editorials.

${ }^{1}$ Climate Change Cluster (C3), University of Technology Sydney, Ultimo, NSW 2007,

Australia
${ }^{2}$ Address correspondence to D. J. Suggett, email david.suggett@uts.edu.au

${ }^{3}$ Wavelength Reef Cruises, 6/43 Macrossan Street, Port Douglas, QLD 4877, Australia ${ }^{4}$ TropWATER, James Cook University, Townsville, QLD 4811, Australia

${ }^{5}$ Program in Fisheries and Aquatic Sciences, School of Forest Resources and Conservation, University of Florida, Gainesville, FL 32653, U.S.A

${ }^{6}$ Center for Conservation, The Florida Aquarium, Apollo Beach, FL 33602, U.S.A.

(C) 2018 Society for Ecological Restoration

doi: 10.1111/rec.12916

Supporting information at:

http://onlinelibrary.wiley.com/doi/10.1111/rec.12916/suppinfo 
et al. 2018), which we term collectively here as coral propagules. Even so, success of these programs is not guaranteed, let alone maximized (Rinkevich 2014; Lirman \& Schopmeyer 2016), particularly where "one size fits all" practices are not tailored to the local taxa and/or reefs in question (Shaver \& Silliman 2017; Ladd et al. 2018) or novel techniques are not systematically evaluated against more established practices. Success for any given practice is gauged as biomass yielded (return-on-effort [RRE]; Rohr et al. 2018) and ultimately underpins cost-effectiveness and scalability of coral reef restoration (Bayraktarov et al. 2016).

Coral nursery and outplanting success to date has almost exclusively been determined from propagule growth and/or survivorship over time (e.g. Lirman \& Schopmeyer 2016; Hein et al. 2017). While these two metrics are not (should not be) the sole measures of success (Bayraktarov et al. 2016, 2017; Hein et al. 2017; Rinkevich 2017), growth and survival are the most easily adopted metrics especially where monitoring capability is limited. Based on these metrics, for any given resource outlay (infrastructure, personnel, and so on), the RRE of coral propagation can be broadly conceptualized by maximizing yield from increasing propagule growth and survival (Fig. 1A). Greatest RRE will result from taxa (species, genotypes) and practices that together promote both enhanced growth and survivorship (Fig. 1A; moving from bottom left to top right). However, life-history dynamics of different coral taxa will inherently preferentially weight RRE to high growth-lower survivorship (e.g. Acropora spp.) versus lower growth-high survivorship (e.g. Porites spp.) (Darling et al. 2012; Madin et al. 2016b), or $r$ versus $K$ selected coral taxa (Sleeman et al. 2005; Montero-Serra et al. 2018). While many nurseries have focused on fast growing species (see Table S1, Supporting Information) to attempt to rapidly boost local coral cover and associated habitat complexity at low cost, propagating slow growing species may be desirable where the goal is to also maintain diversity (Muko \& Iwasa 2011; Montero-Serra et al. 2018). In both cases, overcoming conventional $r$ versus $K$ life-history bottlenecks is likely necessary to maximize success; e.g. fusion of slow growing massive coral micro-fragments (Forsman et al. 2015) or selecting for genotypes of fast growing Acropora spp. that have enhanced stress tolerance, and hence survivorship (Ladd et al. 2017; Lohr \& Patterson 2017; Goergen \& Gilliam 2018). Ultimately a balance of different life histories and morphologies may be required to restore reef sites depending on the goal (Muko \& Iwasa 2011; Ladd et al. 2018), which would thus require disproportionate propagation effort across coral taxa.

Many coral nurseries established to aid recovery of reefs impacted by broadscale ecological impacts, such as heat waves (e.g. Ladd et al. 2017), disease outbreaks (Lirman \& Schopmeyer 2016), or urbanization (e.g. Afiq-Rosli et al. 2017; Chou et al. 2017), now simultaneously propagate multiple taxa with different life-history traits that preferentially select growth or survivorship. Time and effort spent maintaining nurseries and subsequent outplanting introduces a major hurdle to the cost-effectiveness of reef restoration (e.g. Rinkevich 2014; Bayraktarov et al. 2016; Chamberland et al. 2017). Consequently, identifying the optimal proportion of propagules from multiple taxa that can be grown/outplanted simultaneously, while maximizing the yield from all taxa, is a key step towards minimizing costs and inefficiencies of propagation. However, such knowledge for any given nursery or outplanting practice remains largely subjectively derived from past successes and failures. This is particularly problematic for locations where no local history of reef restoration exists with which to guide best practice, such as the Great Barrier Reef (GBR). While early coral restoration efforts were largely developed through trial and error, more recent systematic and quantitative evaluation of practice is growing a wealth of data that can potentially inform coral restoration operations in areas where no local history exists (Boström-Einarsson et al. 2018).

In recently establishing the first multi-species coral nursery on the GBR (Opal Reef, February 2018), we initially considered fundamental questions that determined our RRE. Specifically, of the local GBR coral taxa most desirable to propagate, which (1) have inherently lower survivorship but higher growth that require disproportionately high effort to propagate (including high nursery space requirements)? and (2) have higher survivorship but lower growth and hence require less effort but greater time investment to propagate? To address these unknowns, we developed a relative RRE scoring criterion based on growth versus survivorship from existing data of nursery and outplanted corals to date worldwide; this criterion is termed relative since the RRE score does not account for the resource outlay (e.g. infrastructure and personnel costs) associated with the effort used. We first use the RRE score criterion to identify differences in RRE for different growth morphs and/or genera from restoration practices to date. We then provide a case study from Opal Reef demonstrating how this criterion can be applied, discussing how it provides a novel tool to aid decision-making that could guide future propagation (and outplanting) efforts.

\section{Methods}

\section{Development of RRE Score}

We conducted a literature review using the terms "coral and nursery" or "coral and restoration" to retrieve all nursery and outplanting studies that had recorded concurrent metrics of growth and survivorship, that is measures of growth from the proportion of surviving propagules at any given time (ISI Web Of Science, Thompson Scientific; May 18, 2018). All publications retrieved were cross referred with a global database of coral restoration case studies (Boström-Einarsson et al. 2018) that encompassed a wide range of data sources less likely to be catalogued by Web of Science (i.e. theses and government reports). Studies were only included if they contained both start and end propagule sizes, as well as survivorship, to ensure consistency in data extraction. Data were categorized as nursery propagule, outplant propagule (from in situ nursery or ex situ cultivation), or transplant (in situ movement of wild types). Ex situ adult and juvenile datasets were excluded since our goals were to purely evaluate in situ practices. However, studies of young propagules deployed on the reef initially reared via sexual propagation onto plugs (e.g. Toh et al. 2014) or tetrapods 
(e.g. Chamberland et al. 2017) through ex situ practices were included and labeled as "juvenile"; these "juvenile" data only comprised approximately $10 \%$ of the total dataset. Other metrics recorded were location, species, and growth morph (following coral-trait database; Madin et al. 2016a), attachment method, depth (m), and time of propagation (months). Together these criteria resulted in a total of 52 studies out of 121 papers, yielding 246 data points (as detailed in Table S1). The final dataset spanned papers published from 1995 to 2018, and consisted of 56 species (within 25 genera), of which approximately 60\% were Acropora spp. (104 data, 19 species) and Pocillopora spp. and Stylophora spp. (43 data, six species).

Coral size (and hence growth) was typically reported in three ways across studies, propagule (nubbin) length, or total linear extension (TLE, $\mathrm{cm}$ ) for branching thicket species of Acropora (e.g. Lirman et al. 2014; Lohr et al. 2015), colony area $\left(\mathrm{cm}^{2}\right)$, or ecological volume $\left(\mathrm{cm}^{3}\right.$, e.g. Toh et al. 2014 , and references therein). Since these metrics all capture changing size suitable to describe competitive fitness for space of these different growth morphs, we did not standardize size units. Instead, relative growth was reported as \% change in size normalized to time (months), and hence with units $\% / m o n t h$ as,

$$
\% \text { Growth }=\left(\left[\Delta G_{(t 2-t 1)} /\left(t_{2}-t_{1}\right)\right] / \mathrm{G}_{\mathrm{t} 1}\right) \times 100
$$

where $G$ is size $\left(\mathrm{cm}, \mathrm{cm}^{2}\right.$, or $\left.\mathrm{cm}^{3}\right)$ and $t_{1}$ and $t_{2}$ are the study start and end months, respectively. Prior to examining \% Growth (Eq. 1) versus \% Survivorship data, all percentage data were initially tested for normality (Shapiro-Wilk) and subsequently arcsine (\% Survivorship/100) or Ln transformed (\% Growth) to stabilize variance (MATLAB R2018a, The Mathworks Inc., Natick, MA, U.S.A.). Survivorship values are bounded between $0 \%$ and $100 \%$ (or arcsine (\%Survivorship/100), $0-1.57)$, whereas values of $\%$ Growth had no upper bound. However, values of $\mathrm{Ln}$ (\% Growth) fell within the range -2 to +8 , and thus the few studies recording no growth were assigned an arbitrary lower bound value of -2 . It should be noted that Ln (\% Growth) values of $7-8$ were few $(<1 \%$ of all data) and represent very large growth rates of $>1,000 \% /$ month. While future data could conceivably exceed such rates, the probability is likely extremely rare and therefore the upper bound of +8 was applied to represent a "maximum success" for \% Growth.

To quantify RRE, we first assigned each of transformed $\%$ Growth $(-2$ to +8$)$ and \% Survivorship (0-1.571) into 10 equal divisions to yield 100 sectors (Fig. 1C). Each sector was increasingly scored against growth and survivorship. For example, $0-0.157 \%$ Survivorship and -2 to $-1 \%$ Growth was scored 2 (i.e. $1+1$ ), $0.157-0.314 \%$ Survivorship and -2 to $-1 \%$ was scored 3 (i.e. $2+1$ ), and so on, thereby providing a "relative RRE" score that systematically increases from bottom left to top right. In this way, the interactive nature of changes to growth versus survivorship can be visualized within the RRE scoring framework (Fig. S1). Each of the 246 data points from across all taxa was finally assigned the score based on the sector it fell within (Fig. 1B-D) for subsequent analysis.

\section{GBR Nursery}

In February 2018, we established the first multi-species coral nursery on the GBR at Opal Reef $\left(16^{\circ} 13^{\prime} \mathrm{S} 145^{\circ} 53.5^{\prime} \mathrm{E}\right.$, $24.7 \mathrm{~km}^{2}$ ), which is a "moderate use reef" from "high intensity tourism" (Spalding et al. 2017) via 16 commercial moorings. Opal Reef is one of the most accessible outer GBR sites for tourism, but was heavily impacted by the 2016-2017 El Niño heat wave events where more than $50 \%$ coral cover was lost through bleaching-induced mortality (AIMS 2018) in a highly patchy manner across the reef (J. Edmondson 2018, personal observation). As such, Opal Reef is of high value to the tourism economy on the GBR and hence considered a key site for localized and targeted coral nursey-based reef restoration.

As of August 2018, the Opal Reef nursery consisted of 16 nursery platforms deployed at two reef sites spaced $2.2 \mathrm{~km}$ apart, at 5-6 $\mathrm{m}$ depth on sandy lagoons adjacent to the reef edge. Each platform consisted of $2.0 \times 1.2 \mathrm{~m}$ aluminium diamond-mesh held in place with $2 \times 9 \mathrm{~kg}$ weights and a $20 \mathrm{~L}$ float (see Fig. S2). In this way, the platforms were suspended approximately 1-2 $\mathrm{m}$ above sand. Platforms were first deployed at the first site in February-June 2018 and then at the second site in August-September 2018, to house a total of 2,536 propagules (1,440 and 1,096 at the two sites, respectively) from 11 coral species: Acropora humilis, Acropora hyacinthus, Acropora intermedia, Acropora loripes, Acropora millipora, Acropora tenuis, Montipora hispada, Montipora spumosa, Pocillopora verrucosa, Porites cylindrica, and Turbinaria reniformis. These various species were selected as they were found in sufficient numbers locally to demonstrate survival through the 2016-2017 heat waves (either through environmental refuge or stress resistance, sensu Camp et al. 2018), while also including species with different stress susceptibilities and growth morphologies (Darling et al. 2012; Hoogenboom et al. 2017; Hughes et al. 2018). All propagules were sourced from colonies at both sites. With the exception of A. loripes, T. reniformis, $M$. hispada, and $M$. spumosa, these species were present in previous reef restoration studies used in the development of the RRE scoring (Table S1). All coral nursery activities were conducted under a Great Barrier Reef Marine Park Authority permit (G18/40023.1).

Colonies were collected by hand (hammer and chisel) at 3-5 $\mathrm{m}$ depth, and brought to our operations vessel (Wavelength 5) for immediate fragmentation. Each individual colony yielded 5-100 propagules ranging $0.33-91.2 \mathrm{~cm}^{2}$ surface area (length $\times$ height), with approximately one-third of all propagules in size class bins of less than 6, 6-14, and greater than $14 \mathrm{~cm}^{2}$. All surface area measurements were determined through image analysis (ImageJ2; Rueden et al. 2017) of scaled photographs (TG (Tough)-5; Olympus Australia Pty. Ltd., Sydney). Each propagule was placed on a small $(2 \times 2 \mathrm{~cm})$ travertine tile for attachment to the aluminum mesh frame using cable ties, ensuring the propagule would fuse to the tile and not the nursery frame for later outplanting. No manual cleaning of the aluminum frames was required since platforms remained largely algal free via grazing by herbivorous fish from adjacent coral outcrops. All platforms were monitored on a regular basis 
(A)

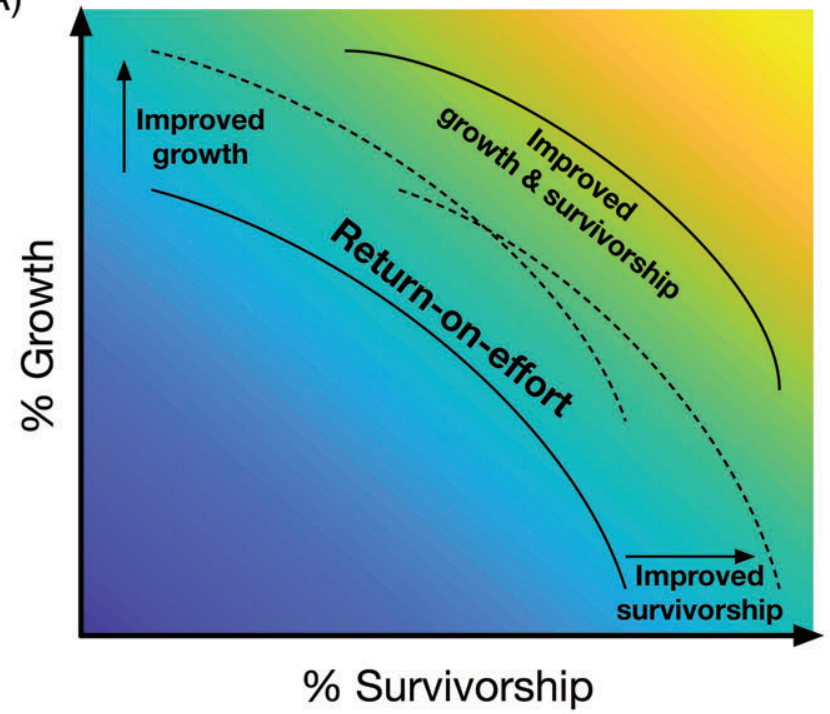

(C)

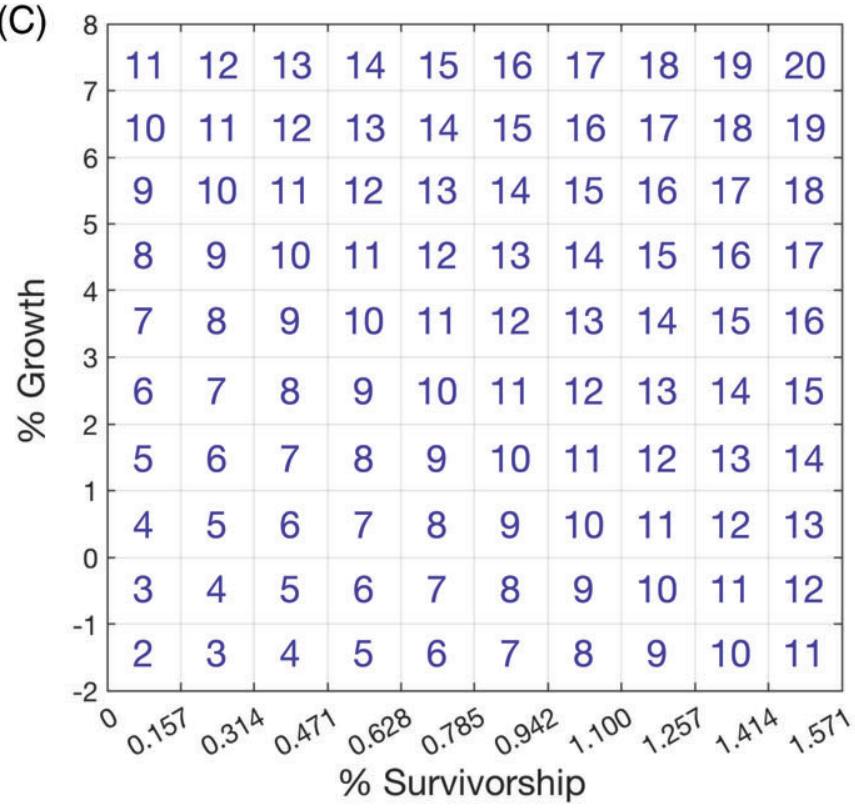

(B)

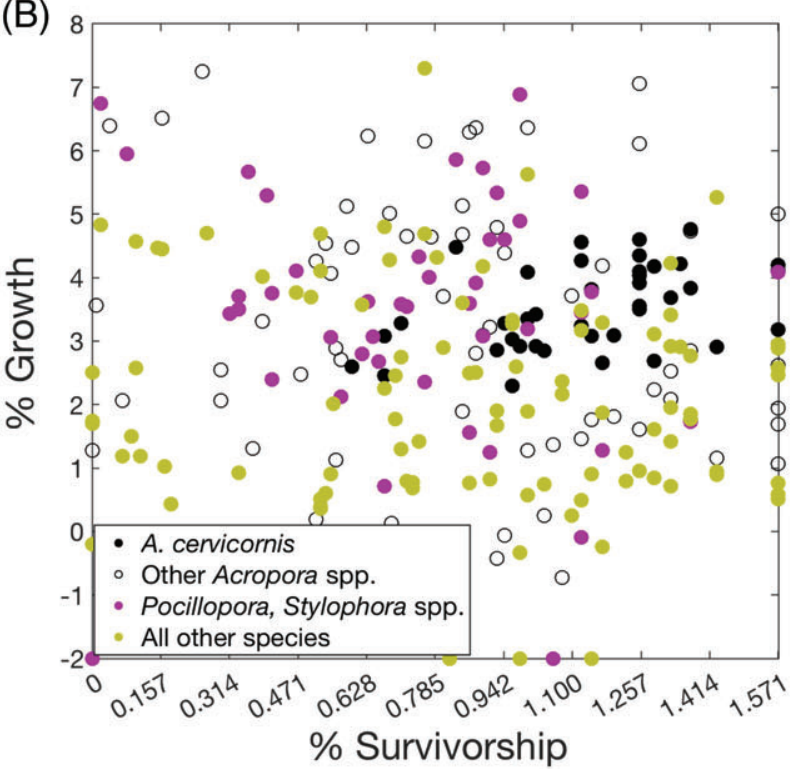

(D)
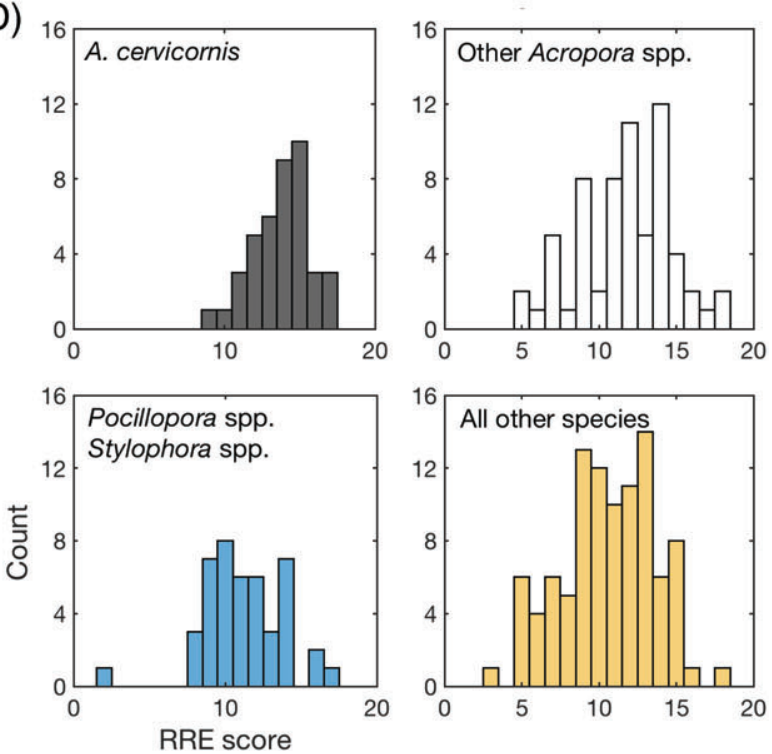

Figure 1. (A) Schematic interaction of changes in growth versus survivorship of coral propagules used to gauge "success" (return-on-effort) of reef restoration activities (nurseries, outplants, and transplants). Increased growth or survivorship can equally enhance return-on-effort relative to the specific resource (costs) outlay (dashed line). Greatest increase to "success" comes from increasing both growth and survivorship—moving diagonally from bottom left to top right. Ultimately, a minimum threshold of return-on-effort will exist to justify cost and/or ecological impact of harvesting and/or attempting propagation (solid lines). Practices must enhance growth and/or survivorship for viability; (B) transformed values of \% Growth versus \% Survivorship of all data extracted from relevant nursery, outplanting, and transplanting studies to date (see main text). Data is binned into the four largest coral taxa categories: Acropora cervicornis (the single most propagated species), "Other" Acropora spp., Pocillopora and Stylophora spp., and "All other" species; (C) relative return-on-effort (RRE) scoring criteria applied to the \% Growth versus \% Survivorship landscape. Values increase linearly with both (transformed) values of $\%$ Growth and \% Survivorship, and hence with increased return-on-effort; and (D) size-frequency distributions of RRE values for data in panel B applied to the scoring in panel $\mathrm{C}$.

(1-2 months) to assess survivorship, with any dead propagules immediately removed. Frames from the first site were transiently removed in August for repeat growth measurements of six species (A. humilis, A. loripes, A. tenuis, M. spumosa, $P$. verrucosa, and $T$. reniformis) by scaled photography in preparation for outplanting.

\section{Results}

\section{Taxonomic Variation in Return-on-Effort}

We initially compared transformed values of $\%$ Growth versus $\%$ Survivorship from literature-extracted data that was binned into the four largest taxonomic groupings, Acropora cervicornis 
( $n=41)$, all other Acropora spp. ( $n=64)$, Pocillopora and Stylophora spp. $(n=43)$, and all other species $(n=98)$ (Fig. 1B). As expected, data from all of these categories was highly scattered with no clear pattern, presumably reflecting study-specific differences in local environmental conditions, and/or the influence of additional confounding factors that regulate growth and survivorship.

Previous studies have suggested growth over time may not be linear but instead depend on duration within nursery and/or initial propagule size (Okubo et al. 2005; Lirman et al. 2014; dela Cruz et al. 2015; Pausch et al. 2018), in particular "juveniles" (Toh et al. 2014). Therefore, in order to check for possible bias from such factors, we first evaluated whether our extracted dataset was affected by duration within nursery/outplant or initial propagule size for the three main species data groupings (Acropora spp., Pocillopora and Stylophora spp., and all other taxa; Figs. S3 \& S4). Overall, no clear trend was evident for either \% Growth (Eq. 1) or \% Survivorship with duration for any of the three species groupings (analysis of variance [ANOVA] and subsequent post hoc analysis, Table S2). In contrast, while $\%$ Survivorship exhibited no clear pattern with initial propagule size, \% Growth decreased with increasing initial propagule size for all three species groupings (Fig. S4). Thus, propagule size appears to regulate \% Growth but in a manner that remains highly consistent across species groups. We next examined whether the approach used (nursery versus outplant or transplant) also potentially influenced \% Growth or \% Survivorship and hence success. \% Survivorship was not dependent on approach. However, \% Growth was significantly reduced for transplanted compared to nursery-grown or outplanted propagules for both Acropora spp. and "All other" species grouping (Fig. S5 \& Table S3), confirming that nursery propagation may often be important to improve success (Shafir et al. 2006; dela Cruz et al. 2015). As such, we acknowledge that some variation observed within taxonomic groups (Fig. 1B \& 1D) could reflect integrating data from both nursery and outplanting studies.

Initial size-frequency distributions of the RRE scores demonstrated that A. cervicornis and "Other Acropora spp." groupings were generally more negatively skewed (shifted towards higher RRE scores) than both "Pocillopora \& Stylophora spp." and "All other taxa" species groupings (Fig. 1D). Thus Acropora spp. consistently yield greater relative return-on-effort than other coral taxa. We further examined RRE scores for individual coral species ("Global" values of each species across the dataset; Table S4). A substantial number of coral species have now been utilized for nursery and outplanting practices worldwide ( $n=128$ and 58, respectively; Boström-Einarsson et al. 2018; also Rinkevich 2014), and we retrieved an RRE score for 56 species representing $45 \%$ of all species used to date in nursery propagation (Boström-Einarsson et al. 2018). Across our entire dataset we obtained a mean RRE $( \pm \mathrm{SE})$ score of $11.52(0.191)(n=246)$, but with only $40 \%$ of species scoring values higher than this entire dataset score. However, it is important to note that more than half of all species were scored by relatively few data points $(n=1-2)$ from a single study location (Tables S1 \& S4). When only considering the species with comparatively greater data $(n>5)$ from across multiple studies, the highest RRE scores (12.6-13.8) obtained were from A. cervicornis, Acropora hemprichii, Montipora digitata, and Porites cylindrica, and the lowest RRE scores (9.7-10.6) from Acropora hyacinthus, Acropora millepora, Montipora sabricula, Pachyseris speciosa, and Stylophora pistillata.

In order to overcome some of the limitations of few data points per species, we next examined RRE scores for species binned according to morphological group or genus (Fig. 2). Coral morphology is a key trait supporting ecological complexity and biodiversity in reefs (Darling et al. 2012; Madin et al. 2016a, 2016b), and so enhancing morphological diversity is a desired goal for many restoration practices (Sleeman et al. 2005; Montero-Serra et al. 2018). Furthermore, binning by morphology overcomes difficulties in reconciling patterns where species differ across bioregions. Many morphologies ultimately returned the same range of RRE scores (10-14), but with "Acropora Branching Closed" and "Digitate" with the highest $(14.0, n=2)$ and lowest $(6.3 \pm 1.8, n=3) \mathrm{RRE}$ scores, respectively (Fig. 2A \& 2B). RRE scores for "Acropora Branching Open" $(12.9 \pm 0.3, n=75)$, "Massive" and "Laminar" $(9.9 \pm 0.4, n=27 ; 9.8 \pm 5.3, n=35)$, and "Digitate" were statistically different from one another (ANOVA; see Fig. 2). However, "Acropora Branching Open" was statistically inseparable from "Acropora corymbose," "Branching Closed," and "Branching Open." Overall the morphology-based RRE scores indicate generally greater success for branching compared to massive taxa. When binned according to genus (Fig. 2C \& 2D), Acropora spp., Hydnophora spp., Montipora spp., and Podabacia spp. had consistently highest RRE scores (approximately 12-13) and Goniastrea spp., Pavona spp., Merulina spp., and Turbinaria spp. consistently lowest RRE scores (approximately 6-9). That said, RRE scores were generally statistically the same across genus, likely reflecting the low number of samples retained when binning by genus (Fig. 2). Interestingly, RRE scores for the main taxonomic groups by genus (i.e. Acropora sp., Pocillopora and Stylophora spp., and "All other" taxa) did not systematically vary over time (Fig. S6) suggesting that RRE variance observed was not driven by improvements in propagation success over time.

\section{Applying RRE Criteria: The Opal Reef Case Study}

Total propagule (or colony) numbers successfully propagated clearly present a major factor in the cost-effectiveness of nursery/outplant practices (e.g. Bayraktarov et al. 2016; Chamberland et al. 2017). However, depending on the restoration goal, not all coral species will necessarily require similar propagule numbers as a result of differences in yields returned from growth and/or survivorship. Therefore, we contrasted our initial propagule (stocking) numbers within the Opal Reef nursery as of August 2018 (Table S5) versus "Global" RRE values returned for the same species (or morphology) (see Fig. 3). This comparison demonstrated that our propagule numbers followed a generally conserved RRE/propagule (Fig. 3A). Specifically, most species clustered around a single trajectory, whereby number of propagules increases as RRE score decreases, and hence 
(A)

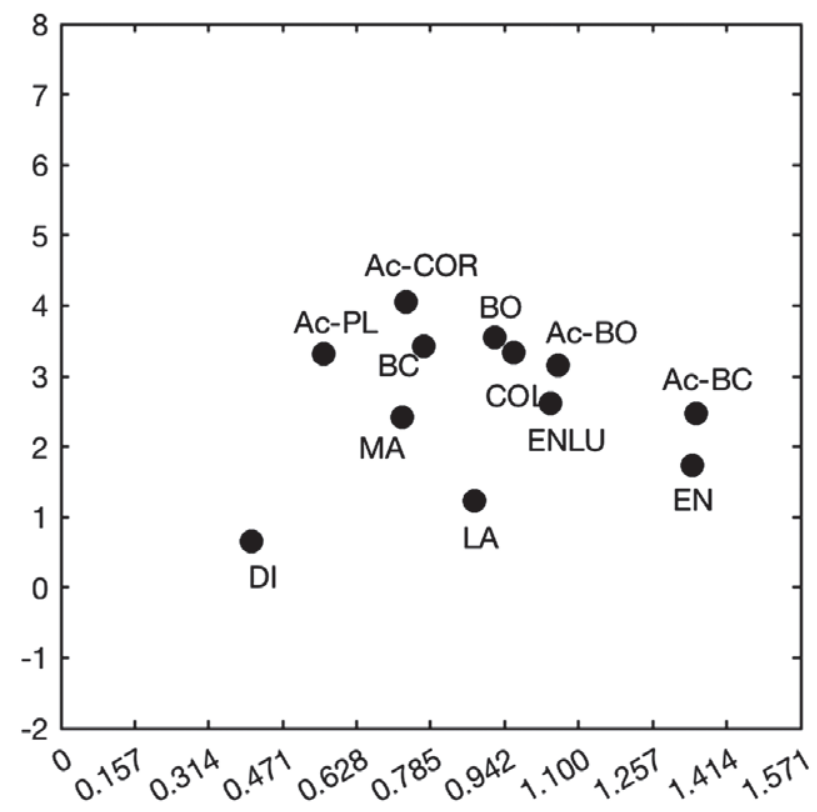

(C)

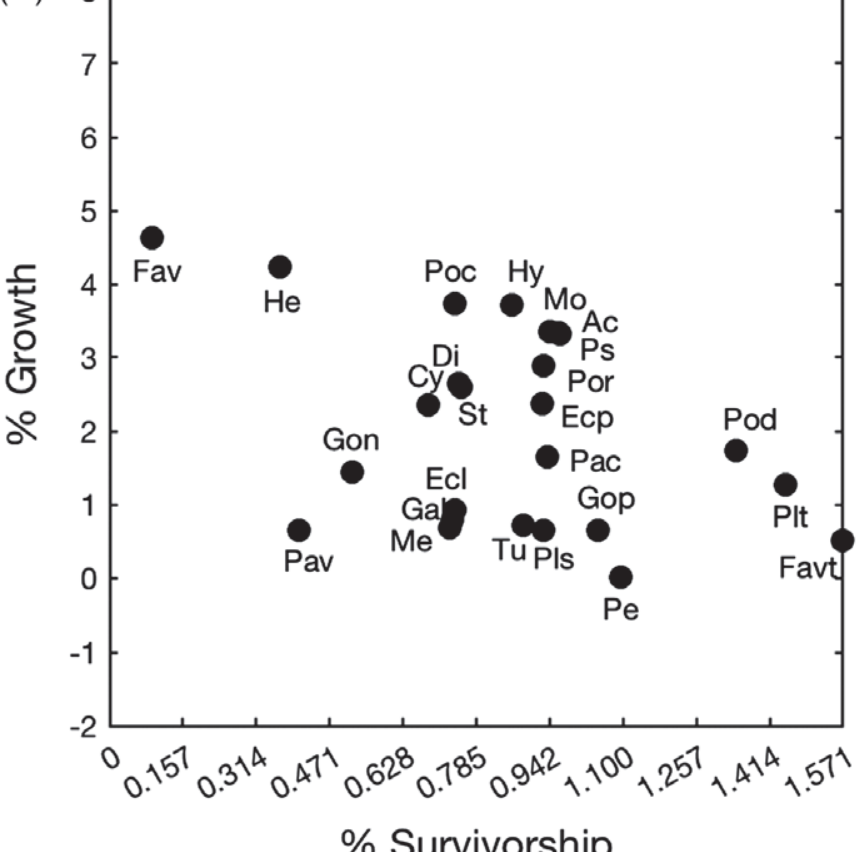

(B)

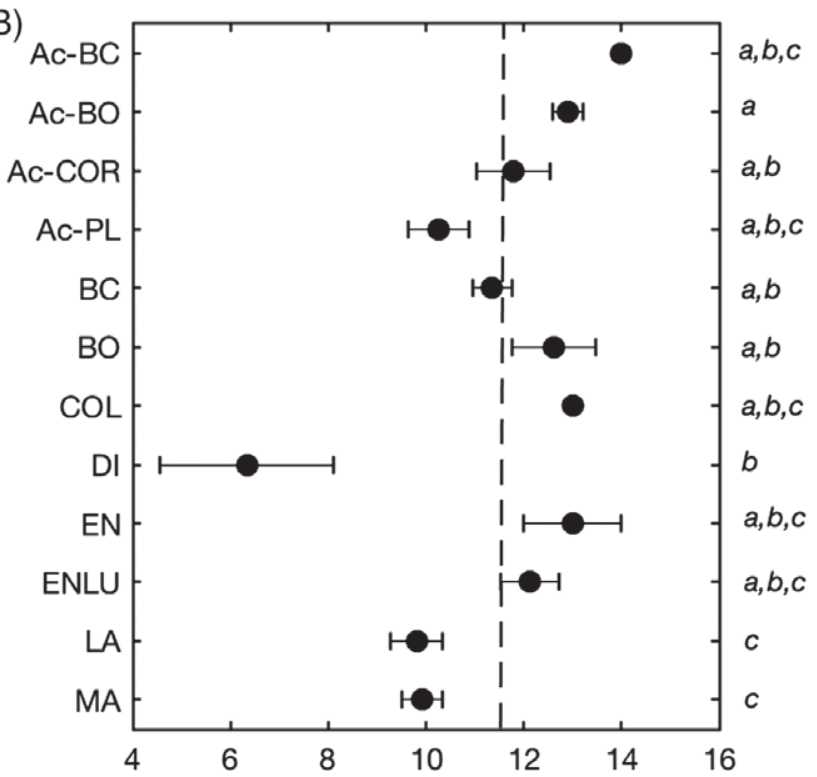

(D)

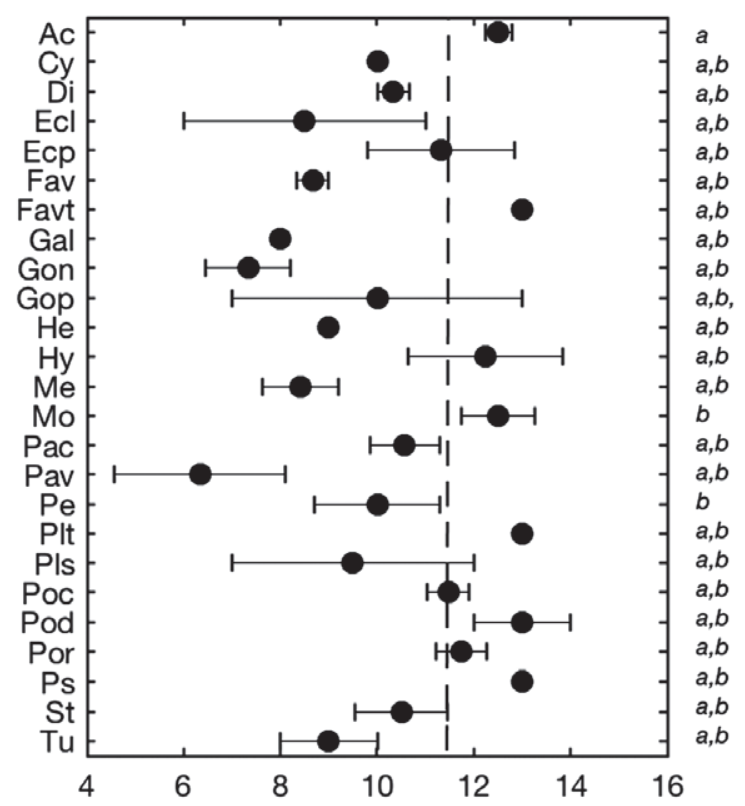

RRE Score

Figure 2. Plots of (transformed) \% Growth (Eq.1) versus \% Survivorship for all data binned by either (A) morphology or (C) genus. Each point represents the mean for each morphology group or genus with SE omitted for clarity; and "global" mean ( \pm SE) relative return-on-effort (RRE) score for all data binned by either (B) morphology or (D) genus; vertical dashed line represents mean RRE score from the entire dataset $(n=246)$. Abbreviations for morphologies are Acropora Branching Closed (Ac-BC), Acropora Branching Open (Ac-BO), Acropora corymbose (Ac-COR), Acropora Plating (Ac-PL), Other Branching Closed (BC), Other Branching Open (BO), Columnar (COL), Digitate (DI), Encrusting (En), Encrusting Long Upright (ENLU), Laminar (LA), Massive (MA) (see main text; also, Table S1 for species bins to morphologies). Abbreviations for genera are: Acropora (Ac), Cyphastrea (Cy), Diploastrea (Di), Echinophyllia (Ecl), Echinopora (Ecp), Favia (Fav), Favites (Favt), Galaxia (Gal), Goniastrea (Gon), Goniopora (Gop), Heliopora (Hel), Hydnophora (Hy), Merulina (Me), Montipora (Mo), Pachyseris (Pac), Pavona (Pav), Pectinia (Pe), Platygyra (Plt), Plesiastrea (Pls), Pocillopora (Poc), Podabacia (Pod), Porites (Por), Psammacora (Ps), Stylophora (St), Turbinaria (Tu). Post hoc Tukey test groupings are shown as italicized letters following ANOVA to test for differences among RRE means for (B) morphologies $(F=5.533, p<0.001)$ and (D) genera $(F=2.797, p<0.001)$. Statistical analysis was conducted using MATLAB R2018a. 

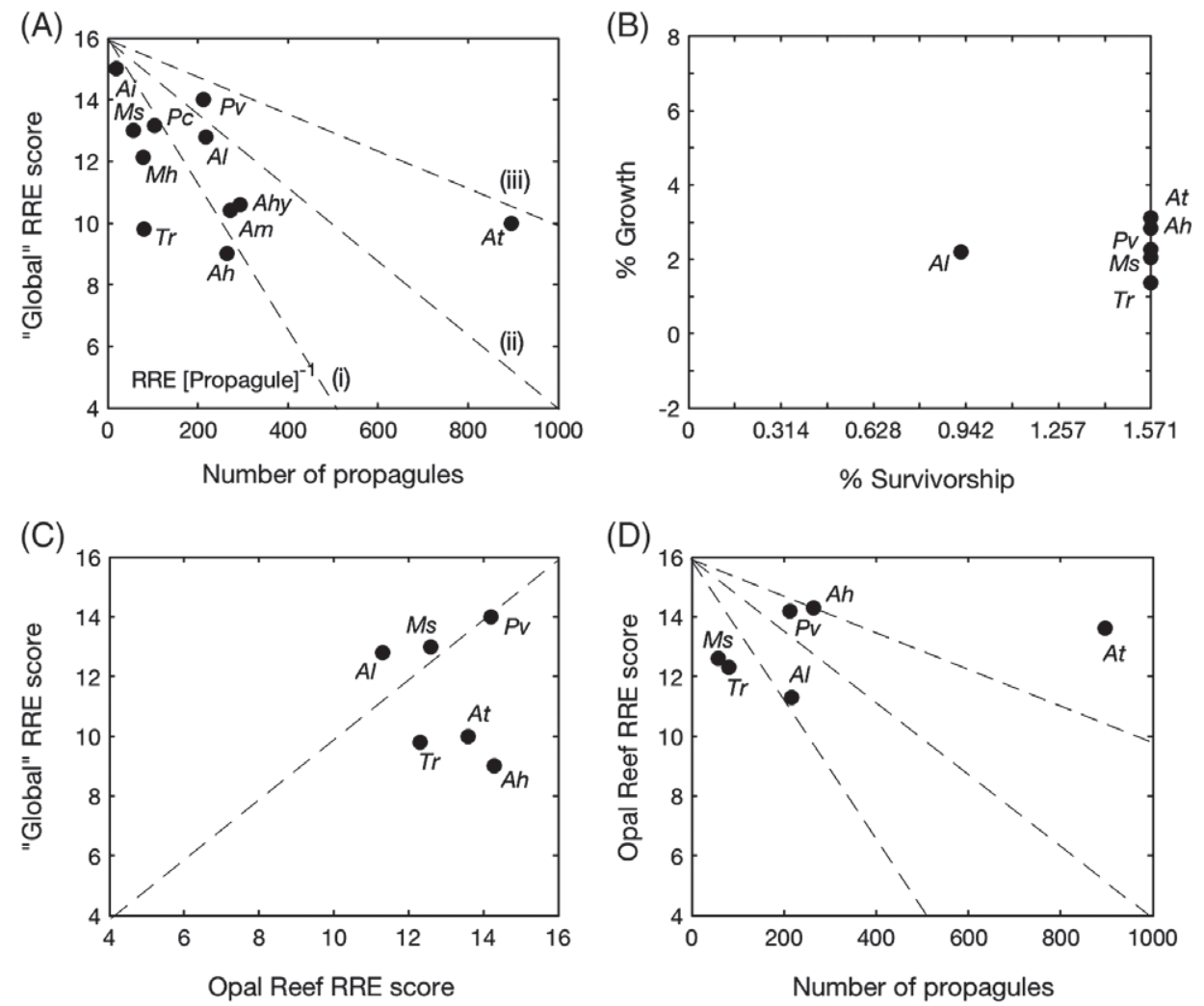

Figure 3. Application of RRE scoring to Opal Reef nursery data through comparison of (A) number of propagules versus "global" RRE score for each coral taxon at Opal Reef (see Table S5): Acropora humilis (Ah), Acropora hyacinthus (Ahy), Acropora intermedia (Ai), Acropora loripes (Al), Acropora millipora (Am), Acropora tenuis (At), Montipora hispada (Mh), Montipora spumosa (Ms), Pocillopora verrucosa (Pv), Porites cylindrica (Pc), Turbinaria reniformis (Tr). Note that "global" RRE scores were available for all taxa except $A l, M s, H h, T r$. RRE scores for morphology were used for these four taxa since genus RRE scores for Montipora spp. were heavily influenced by branching M. digitata (but where $M h$ and $M s$ are "laminar") and for Acropora spp. confounded by multiple morphs (but where $A l$ is "Corymbose"). Diagonal dashed lines indicate trajectories of RRE/propagule that are relatively (i) low, (ii) medium, and (iii) high based on the total number of propagules currently at Opal Reef nursery; (B) transformed values of \% Growth versus \% Survivorship for six species at Opal Reef nursery for which data currently exists (see main text); (C) Opal Reef RRE scores derived from distribution of \% Growth versus \% Survivorship in panel B versus "global" RRE scores, where the diagonal dashed line represents the 1:1; and (D) number of propagules versus RRE score for each coral taxon at Opal Reef. Only mean values are shown throughout for clarity, and all data for Opal Reef is up to August 2018.

value of RRE/propagule. Assuming that the target is to produce equal yields of all taxa, Acropora tenuis therefore appears "over-stocked" within the nursery relative to other taxa, since it falls on a much higher trajectory of RRE/propagule. Similarly, Turbinaria reniformis particularly appears "under-stocked" as a result of a low RRE/propagule trajectory.

RRE scores were determined for six species propagated at Opal Reef (Acropora humilis, Acropora loripes, A. tenuis, Montipora spumosa, Pocillopora verrucosa, and T. reniformis; Table S5) by determining \% Growth and \% Survivorship May-August 2018. Values for transformed \% Growth (Eq. 1) varied between 1.37 and 3.13, and were thus consistent with \% Growth achieved relative to the initial propagule size observed from previous studies (see Fig. S7). With the exception of A. loripes, all species in our nursery retained $100 \%$ survival by August 2018 (Fig. 3B). Consequently, RRE scores varied between 11.3 and 14.3 (Fig. 3B \& Table S5) and with values for A. loripes, P. verrucosa, and Montipora hispada that were similar to their "Global" RRE scores. In contrast,
RRE scores for Opal Reef (and hence RRE/propagule; Fig. 3D) were higher than for the "Global" assessment (Fig. 3C) for A. humilis, A. tenuis, and T. reniformis, suggesting that these three taxa are more successfully propagated at Opal Reef compared to sites/seasons that comprise the "Global" RRE score (see Table S1).

\section{Discussion}

\section{Drivers of Variance Among RRE Scores}

Concurrent measurements of growth and survivorship demonstrate considerable variance in relative RRE achieved through in situ coral propagation practices to date. Restoration practices have been undertaken on several key coral species for at least 20 years, and hence it is plausible to expect an increase in RRE score over time where practices to enhance success have improved. However, no clear change in RRE score over time 
was evident for any species. For example, RRE scores for Acropora cervicornis of 9-16 (e.g. Ross 2012; Hernandez-Delgado et al. 2014) compared to 11-14 (e.g. Schopmeyer et al. 2017; Goergen \& Gilliam 2018), and for Stylophora pistillata RRE scores of 10-12 (Rinkevich 2000; Shafir et al. 2006) compared to 2-14 (Kotb 2016; Rachmilovitz \& Rinkevich 2017). Identifying any potential improvement in practices over time is likely confounded by integrating datasets with highly variable RRE scores retrieved from differences in success from local conditions and genotypes.

Generally high RRE scores were returned from Acropora spp. In the case of $A$. cervicornis, these scores were typically from a limited range of \% Growth (approximately 2-5) but generally high range of $\%$ Survivorship (approximately $>0.628$ ), presumably reflecting the popularity of the "tree" culture method (Nedimyer et al. 2011) commonly employed for this species, which promotes rapid growth across A. cervicornis genotypes (O'Donnell et al. 2017). In addition, large-scale mortality events in A. cervicornis nurseries can be sudden and sporadic (e.g. bleaching events, O'Donnell et al. 2017), and generally not captured in studies monitoring survival over shorter time scales. In contrast, the higher RRE scores for "Other Acropora spp." were weighted by both higher \% Growth (lower \% Survivorship) or higher \% Survivorship (lower \% Growth), likely as a result of different Acropora spp. life-history dynamics and growth conditions (Sleeman et al. 2005; Darling et al. 2012). Such differences within a single genus highlight the uncertainty in yields introduced by adopting a "one size fits all" approach to restoration practices, and the need to consider the specific restoration goals (and resources available) in choosing the most appropriate taxa to propagate.

Lower RRE scores and hence propagation success returned for "Massive" taxa appears to be driven by both reduced \% Growth and \% Survivorship, and thus at odds with their typically $K$-selected life-history strategy (e.g. Darling et al. 2012). Propagation practices may therefore be perceived as particularly sub-optimal for massive taxa and thus require greater effort to successfully increase biomass yields. However, it is important to note that the "Massive" morphology represents a composite from genera with both high (e.g. Porites spp.) and low (e.g. Favia spp., Goniastrea spp., and others) RRE scores, presumably since not all $K$-selected histories are the same (Darling et al. 2012). Different RRE scores for alternate morphologies of Acropora similarly demonstrates that not all $r$-selected life histories are equal (see also Sleeman et al. 2005; Benjamin et al. 2017). Values for both \% Growth and \% Survivorship were highly variable among morphologies of Acropora, resulting in RRE scores ranging from approximately 10-14. Consequently, a single RRE score is insufficient to consider propagation success for Acropora as a genus, not only as a result of competition between growth forms of Acropora (Benjamin et al. 2017) but also inherent differences in stress susceptibility (Hoogenboom et al. 2017). For example, within the genus Acropora, "Acropora plating" (largely Acropora hyacinthus) and "Acropora Branching Open" had lower and higher RRE scores, respectively. Both of these Acropora morphologies were particularly impacted by the 2016-2017 El Niño on the GBR (Hughes et al. 2018), suggesting that effort would need to be disproportionately focused on plating Acropora spp. to yield equivalent success in propagation and/or restoration. Thus, RRE scores need to be considered at both functional (i.e. morphological) and taxonomic levels.

Interestingly, we observed no significant negative correlation between \% Growth and \% Survivorship (for data binned either by morphology or genus), a trend expected where corals inherently trade off resources between growth and physiological maintenance (survivorship) (Darling et al. 2012); such trade-offs are similarly expected for the same coral taxa but growing under different environments (e.g. A. hyacinthus, Bay \& Palumbi 2017). Our outcome thus seems contrary to that reported by Montero-Serra et al. (2018) who recently considered coral transplant studies. These authors identified that survival after transplantation was significantly negatively correlated with mean growth rates measured under natural conditions. However, our contrasting results are likely explained by one or more factors: (1) growth of transplants may not always accurately reflect that of "natural" colonies (as per Montero-Serra et al. 2018); (2) limited sample sizes for some taxa (or morphologies) in our assessment (e.g. "Digitate" or Pavona spp.) do not accurately capture general life-history trade-offs; and/or (3) our approach that collates nursery-grown (and outplanted) propagules as well as transplants confounds how coral taxa are differentially impacted by fragmentation and the conditions imposed through nursery rearing practices (dela Cruz et al. 2015). In the latter case, an RRE score based on past successes and failures (as opposed to gleaning from ecological processes, e.g. Ladd et al. 2018) would therefore seem essential in guiding future practices.

Importantly, the relative propagule densities of different taxa at Opal Reef reflected a particular desire to propagate more bleaching-tolerant species of Acropora, but also consider the potential for "laminar" and "Encrusting" coral species that were less impacted than Acropora spp. during the 2016-2017 heat wave (Hughes et al. 2018). Of all Acropora spp. commonly found at Opal Reef, Acropora tenuis had among the lowest reported bleaching on the GBR during the 2016-2017 heat wave (Hoogenboom et al. 2017). In fact, the RRE/propagule may actually be an underestimate for Opal Reef since the "Global" RRE values for A. tenuis were derived from only two studies, whereby the RRE was much lower as a result of heavily reduced \% Survivorship but similar \% Growth for early juveniles (RRE = 7; dela Cruz \& Harrison 2017) compared to young adults ( $R R E=13$; Omori et al. 2007). It is also unclear whether the low RRE/propagule for the "laminar" Turbinaria reniformis (and the two "Encrusting" species of Montipora: Montipora hispada and Montipora spumosa) reflects an underestimate in the "Global" RRE scores for these morphologies given that the specific taxa used to generate these scores (Turbinaria patula, Montipora aequituberculata) were not the same as those cultivated at Opal Reef. Together, this demonstrates that the "Global" RRE scores provide a means to consider initial propagule densities relative to the desired yields of alternate taxa. However, ultimately site-specific RRE scores are required to fine-tune "success" given the specific species and site conditions in question. 
All colonies used for propagation at Opal reef were large and clearly survived the 2016-2017 heat wave, but it is most likely that such survival was achieved through local heat stress refuges (Hoogenboom et al. 2017; Hughes et al. 2018) compared to genotypes that have been selected with higher heat tolerance (Camp et al. 2018). It is important to note that our current data was only collected from the coolest months, and whether warmer waters would promote factors that reduce survivorship (e.g. enhanced algal growth, disease, and so on) remains to be tested. Even so, any reduced \% Survivorship in warmer months would presumably be offset by enhanced growth expected in nurseries (Xin et al. 2016). Precisely understanding (and documenting) the specific environmental conditions from which RRE scores are determined, and how they vary with season, is therefore clearly essential to pinpoint the dynamic range of RRE scores both locally and globally. Based on our initial assessment for Opal Reef during winter months, RRE scores retrieved would suggest current propagule numbers for Acropora loripes, M. spumosa, and T. reniformis are low compared to other taxa, assuming a goal of achieving equivalent yields for all six taxa.

\section{Future Application of RRE Scoring to Optimize Return-on-Effort}

Evaluating nursery (outplant, transplant) practice success based on RRE scoring both of past data and through initial application to Opal Reef highlights a key component of the RRE as a decision-making tool; specifically, that RRE scoring needs to be iterative, dynamic, and targeted in order to support the decision-making process. RRE scores returned through our data mining of previous studies capture an integrated ("global") view of success from practices reported to date. Such practices often appear to yield high survivorship (approximately $>70 \%$ ) and moderate growth (approximately 25\%/month) (Bayraktarov et al. 2016; Hein et al. 2017), and hence why RRE scores for individual taxa (morphologies) exhibit somewhat limited variance around our RRE dataset mean. RRE scores integrated from across studies are therefore perhaps unlikely to reflect those expected for any specific study (site, practice). This is not to say that these "global" RRE scores are redundant-far from it, since they enable key questions to be framed in initiating any restoration practice: e.g. Is the "global" RRE score high enough to justify the initial resource outlay available for any given taxa? and What RRE score is needed beyond the "global" value to justify the cost (and can practices be put in place to reach this score)? As such, the "global" RRE score provides a dynamic benchmark that will inevitably change over time as more studies return useable data.

Locally operationalizing RRE scores will enable restoration practitioners to better understand which coral taxa are most successfully propagated, when, and where. While this is achieved by contrasting local with "global" RRE scores, identifying how local RRE scores vary over time, and hence the local dynamic range for any given taxa based on site-specific environmental and genotype variation, will be key. However, implementing RRE scoring as a broad means to gauge restoration practice success (in terms of coral propagation) of course inevitably requires standardized practices for data collection and reporting (Hein et al. 2017; Boström-Einarsson et al. 2018), and in the case of "global" RRE scores, a single platform for depositing and viewing RRE data. As we demonstrated, less than 50\% of all possible studies had useable data for producing RRE scores (see Methods section), largely through inconsistencies in reporting growth. While we attempted to maximize useable data through \% Growth (alongside \% Survivorship), our approach may still fail to fully capture how growth can equate to success. For example, new branches/tissue biomass may regulate how much of a colony can be harvested for restoration use (Mercado-Molina et al. 2016) but may not be accounted for in certain growth metrics (e.g. TLE; Lirman et al. 2014; Lohr et al. 2015). Additional factors may also be important to consider RRE scoring and how it is ultimately implemented:

First, understanding the goal of the restoration practice in question will determine how the RRE score is used to consider practice success in terms of maximizing coral yields. Various coral propagation goals exist, including maximizing growth (size) for establishing sexually reproductive populations (dela Cruz \& Harrison 2017), selecting more stress-resistant genotypes (Ladd et al. 2017; Lohr \& Patterson 2017; Goergen \& Gilliam 2018), and/or propagation of fast growing stress-sensitive taxa in environments that may offer refuge from stressors (Shaver \& Silliman 2017). However, the same RRE score can be achieved from low growth-high survivorship and vice versa, potentially confounding the interpretation. The RRE score is therefore useful to benchmark overall yield, but potentially masks the nature by which the score is reached (or must be improved). Visualizing how the score is attained overcomes this problem, and therefore is as important as the score itself to aid the decision-making process to ascertain (improve) growth versus survival.

Second, propagation that establishes large (relatively slower growing) colonies that are sexually reproductive could be perceived as highly successful for restoration (dela Cruz \& Harrison 2017), yet these colonies would currently return low(er) RRE scores. While incorporating additional criteria into RRE scoring (e.g. size to reproduction, fecundity) could perhaps overcome this problem, it would inevitably introduce more complexity in data collection and reduce broad applicability of RRE scoring.

Third, fully quantifying the resource outlay (and site values) with which the RRE is considered against is needed to return the "absolute return-on-effort". This in turn determines whether any given practice (or taxon) is economically justifiable compared to the asset value of the site in question (Bayraktarov et al. 2016). For example, a relatively low RRE score could be justified where the local asset value is disproportionately high (e.g. through tourism: Spalding et al. 2017; obligatory subsistence dependency: Hein et al. 2017) yet nursery and propagation costs low (e.g. Young et al. 2012). Embedding RRE scoring into cost-effectiveness exercises that are increasingly performed for restoration practices (e.g. Toh et al. 2014; Afiq-Rosli et al. 2017; Chamberland et al. 2017) is therefore a logical step. Similarly, cost analyses could be used to specify RRE-cost thresholds 
and hence set the minimum RRE (and hence yield) with which practices are considered viable.

Establishing broad metrics to evaluate and contrast nursery and outplant success is a key step towards predicting how scalable restoration practices are over space and time. Our novel RRE scoring approach provides practitioners with such a means to benchmark short term success of nursery-reared or transplanted propagules. Persistent differences in reporting growth, in concert with practitioner focus on relatively few species, demonstrates that many species (genera or morphologies) are still under-represented in "global" RRE scores. While data to inform many local practices is therefore still lacking, broad adoption of RRE scoring would overcome this limitation. Applying this scoring approach to the first multi-species coral nursery on the GBR (Opal reef) provides a first step in demonstrating how RRE scoring can inform decision-making processes, but highlights the need to track RRE scores over time to establish how they respond dynamically. Similarly, tracking how RRE scores change as practices span from nursery to outplanting phases is ultimately needed to fully benchmark RRE in restoring sites such as Opal Reef.

\section{Acknowledgments}

The authors wish to express immense thanks to three anonymous reviewers as well as to participants of the Great Barrier Reef Restoration Symposium (Cairns, July 2018), where valuable discussions improved the concepts and data evaluation presented here. Also, to the Great Barrier Reef Marine Parks Authority, whose patience and support established the permit for Opal Reef coral nursery and outplanting (G18/40023.1), as well as staff from Wavelength Reef Cruises who together have continuously supported the nursery operations. This work was supported by essential funding through the AMP Foundation (Tomorrow Maker Award to DJS, "Future-proofing the Great Barrier Reef through climate change-resilient super corals"), Advance Queensland Small Business Innovation Research Initiative Boosting Coral Abundance on the Great Barrier Reef ("Solving the bottleneck of reef rehabilitation through boosting coral abundance: Miniaturising and mechanising coral out-planting" to DJS, EC, and JE), and the Australian National Environmental Science Program's Tropical Water Quality Hub (LBE).

\section{LITERATURE CITED}

Afiq-Rosli L, Taira D, Xin LH, Toh TC, Toh KB, Ng CSL, Cabaitan PC, Chou LM, Song T (2017) In situ nurseries enhance coral transplant growth in sedimented waters. Marine Biology Research 13:878-887

AIMS (Australian Institute of Marine Science) (2018) Opal reef. Long-Term Monitoring and Data Centre, AIMS. http://apps.aims.gov.au/reefmonitoring/reef/16025S2 (accessed 13 Sept 2018)

Anthony K, Bay LK, Costanza R, Firn J, Gunn J, Harrison P, et al. (2017) New interventions are needed to save coral reefs. Nature Ecology and Evolution $1: 1420-1422$

Bay RA, Palumbi SR (2017) Transcriptome predictors of coral survival and growth in a highly variable environment. Ecology and Evolution 7:4794-4803
Bayraktarov E, Saunders MI, Abdullah S, Mills M, Beher J, Possingham HP, Mumby PJ, Lovelock CE (2016) The cost and feasibility of marine coastal restoration. Ecological Applications 26:1055-1074

Bayraktarov E, Saunders MI, Mumby PJ, Possingham HP, Abdullah S, Lovelock CE (2017) Response to "Rebutting the inclined analyses on the cost-effectiveness and feasibility of coral reef restoration". Ecological Applications 27:1974-1980

Benjamin CS, Punongbayan AT, dela Cruz DW, Villanueva RD, Baria MVB, Yap HT (2017) Use of Bayesian analysis with individual-based modeling to project outcomes of coral restoration. Restoration Ecology 25:112-122

Boström-Einarsson L, Ceccarelli D, Bayraktarov E, Cook N, Harrison P, Hein M, et al. (2018) Coral restoration in a changing world - a global synthesis of methods and techniques. A report for the Reef Restoration and Adaptation Program, Subproject 1a-Review of existing technologies/pilots and new initiatives

Camp EF, Schoepf V, Suggett DJ (2018) How can "super corals" facilitate global coral reef survival under rapid environmental and climatic change? Global Change Biology 24:2755-2757

Chamberland VF, Petersen D, Guest JR, Petersen U, Brittsan M, Vermeij MJA (2017) New seeding approach reduces costs and time to outplant sexually propagated corals for reef restoration. Scientific Reports 7:18076

Chou LM, Toh TC, Ng CSL (2017) Effectiveness of reef restoration in Singapore's rapidly urbanizing coastal environment. International Journal of Environmental Science and Development 8:576-580

Clark S, Edwards AJ (1995) Coral transplantation as an aid to reef rehabilitation: evaluation of a case study in the Maldive Islands. Coral Reefs 14:201-213

Darling ES, Alvarez-Filip L, Oliver TA, McClanahan TR, Côté IM, Bellwood D (2012) Evaluating life-history strategies of reef corals from species traits. Ecology Letters 15:1378-1386

Forsman ZH, Page CA, Toonen RJ, Vaughan D (2015) Growing coral larger and faster: micro-colony-fusion as a strategy for accelerating coral cover. PeerJ 3:e1313

Goergen EA, Gilliam DS (2018) Outplanting technique, host genotype, and site affect the initial success of outplanted Acropora cervicornis. PeerJ 6:e4433

Hein MY, Willis BL, Beeden R, Birtles A (2017) The need for broader ecological and socioeconomic tools to evaluate the effectiveness of coral restoration programs. Restoration Ecology 25:873-883

Hernández-Delgado EA, Mercado-Molina AE, Alejandro-Camis PJ, Candelas-Sánchez F, Fonseca-Miranda JS, González-Ramos CM, et al. (2014) Community-based coral reef rehabilitation in a changing climate: Lessons learned from hurricanes, extreme rainfall, and changing land use impacts. Open Journal of Ecology 4:918-944

Hoogenboom MO, Frank GE, Chase TJ, Jurriaans S, Álvarez-Noriega M, Peterson K, et al. (2017) Environmental drivers of variation in bleaching severity of Acropora species during an extreme thermal anomaly. Frontiers in Marine Science 4:376

Hughes TP, Kerry JT, Baird AH, ConnollySR Dietzel A, Eakin CM, Heron SF, et al. (2018) Global warming transforms coral reef assemblages. Nature 556:492-496

Kotb MMA (2016) Coral translocation and farming as mitigation and conservation measures for coastal development in the Red Sea: Aqaba case study, Jordan. Environmental Earth Sciences 75:439

dela Cruz DW, Harrison PL (2017) Enhanced larval supply and recruitment can replenish reef corals on degraded reefs. Scientific Reports 7:13985

dela Cruz DW, Rinkevich B, Gomez ED, Yap HT (2015) Assessing an abridged nursery phase for slow growing corals used in coral restoration. Ecological Engineering 84:408-415

Ladd MC, Shantz AA, Bartels E, Burkepile DE (2017) Thermal stress reveals a genotype-specific tradeoff between growth and tissue loss in restored Acropora cervicornis. Marine Ecology Progress Series 572:129-139

Ladd MC, Miller MW, Hunt JH, Sharp WC, Burkepile DE (2018) Harnessing ecological processes to facilitate coral restoration. Frontiers in Ecology and the Environment 16:239-246

Lirman D, Schopmeyer S (2016) Ecological solutions to reef degradation: optimizing coral reef restoration in the Caribbean and Western Atlantic. PeerJ 4:e2597 
Lirman D, Schopmeyer S, Galvan V, Drury C, Baker AC, Baums IB (2014) Growth dynamics of the threatened Caribbean staghorn coral Acropora cervicornis: influence of host genotype, symbiont identity, colony size, and environmental setting. PLoS One 9:e107253

Lohr KE, Patterson JT (2017) Intraspecific variation in phenotype among nursery-reared staghorn coral Acropora cervicornis (Lamarck, 1816). Journal of Experimental Marine Biology and Ecology 486:87-92

Lohr KE, Bejarano S, Lirman D, Schopmeyer S, Manfrino C (2015) Optimizing the productivity of a coral nursery focused on staghorn coral Acropora cervicornis. Endangered Species Research 27:243-250

Madin JS, Anderson KD, Andreasen Magnus Heide, Bridge Tom CL, Cairns Stephen D, Connolly Sean R, et al. (2016a) The Coral Trait Database, a curated database of trait information for coral species from the global oceans. Scientific Data 3:160017

Madin JS, Hoogenboom MO, Connolly SR, Darling ES, Falster DS, Huang D, et al. (2016b) A trait-based approach to advance coral reef science. Trends in Ecology \& Evolution 31:419-428

Mercado-Molina A, Ruiz-Diaz C, Sabat A (2016) Branching dynamics of transplanted colonies of the threatened coral Acropora cervicornis: morphogenesis, complexity, and modelling. Journal of Experimental Marine Biology and Ecology 482:134-141

Montero-Serra I, Garrabou J, Doak DF, Figuerola L, Hereu B, Ledoux J-B, Linares C (2018) Accounting for life-history strategies and timescales in marine restoration. Conservation Letters 11:1-9

Muko S, Iwasa Y (2011) Long-term effect of coral transplantation: restoration goals and the choice of species. Journal of Theoretical Biology 280:127-138

Nedimyer K, Gaines K, Roach S (2011) Coral Tree Nursery@: an innovative approach to growing corals in an ocean-based field nursery. AACL Bioflux 4:442-446

O’Donnell KE, Lohr KE, Bartels E, Patterson JT (2017) Evaluation of staghorn coral (Acropora cervicornis, Lamarck 1816) production techniques in an ocean-based nursery with consideration of coral genotype. Journal of Experimental Marine Biology and Ecology 487:53-58

Okubo N, Taniguchi H, Motokawa T (2005) Successful methods for transplanting fragments of Acropora formosa and Acropora hyacinthus. Coral Reefs 24:333-342

Omori M, Iwao K, Tamura M (2007) Growth of transplanted Acropora tenuis 2 years after egg culture. Coral Reefs 24:563

Pausch RE, Williams DE, Miller MW (2018) Impacts of fragment genotype, habitat, and size on outplanted elkhorn coral success under thermal stress. Marine Ecology Progress Series 592:109-117

Rachmilovitz EN, Rinkevich B (2017) Tiling the reef—exploring the first step of an ecological engineering tool that may promote phase-shift reversals in coral reefs. Ecological Engineering 105:150-161

Rinkevich B (2000) Steps towards the evaluation of coral reef restoration by using small branch fragments. Marine Biology 136:807-812

Rinkevich B (2014) Rebuilding coral reefs: does active reef restoration lead to ustainable reefs? Current Opinion in Environmental Sustainability 7:28-36

Rinkevich B (2017) Rebutting the inclined analyses on the cost-effectiveness and feasibility of coral reef restoration. Ecological Applications 27: 1970-1973

Rohr JR, Bernhardt E, Cadotte MW, Clements W (2018) The ecology and economics of restoration: when, what, where, and how to restore ecosystems. Ecology and Society 23:15

Coordinating Editor: Gary Kendrick
Ross A (2012) The decline and restoration of Acropora cervicornis in Montego Bay: exploring the anthozoics and anthozoculture of A. cervicornis. $\mathrm{PhD}$ Thesis, The University of the West Indies, Kingston, Jamaica

Rueden CT, Schindelin J, Hiner MC, DeZonia BE, Walter AE, Arena ET, Eliceiri KW (2017) ImageJ2: ImageJ for the next generation of scientific image data. BMC Bioinformatics 18:529

Schopmeyer SA, Lirman D, Bartels E, Gilliam DS, Goergen EA, Griffin SP, Johnson ME, Lustic C, Maxwell K, Walter CS (2017) Regional restoration benchmarks for Acropora cervicornis. Coral Reefs 36:1047-1057

Shafir S, Rijn JV, Rinkevich B (2006) Steps in the construction of underwater coral nursery, an essential component in reef restoration acts. Marine Biology 149:679-687

Shaver EC, Silliman BR (2017) Time to cash in on positive interactions for coral restoration. PeerJ 5:e3499

Sleeman JC, Boggs GC, Radford BC, Kendrick GA (2005) Using agent-based models to aid reef restoration: enhancing coral cover and topographic complexity through the spatial arrangement of coral transplants. Restoration Ecology 13:685-694

Spalding M, Burke L, Wood SA, Ashpole J, Hutchinson J, zu Ermgassen P (2017) Mapping the global value and distribution of coral reef tourism. Marine Policy 82:104-117

Toh TC, Ng CSL, Peh JWK, Toh KB, Chou LM (2014) Augmenting the post-transplantation growth and survivorship of juvenile scleractinian corals via nutritional enhancement. PLoS One 9:e98529

Xin LH, Adzis KAA, Hyde J, Cob ZC (2016) Growth performance of Acropora formosa in natural reefs and coral nurseries for reef restoration. AACL Bioflux 9:1090-1100

Young CM, Schopmeyer SA, Lirman DA (2012) A review of reef restoration and coral propagation using the threatened genus Acropora in the Caribbean and western Atlantic. Bulletin of Marine Science 88:1075-1098

\section{Supporting Information}

The following information may be found in the online version of this article:

Table S1. Summary of all literature used for this study.

Table S2. ANOVA and post hoc Tukey tests of transformed values of \% Growth [month $]^{-1}$ (Ln) and \% Survivorship (Arcsin). binned according to time.

Table S3. ANOVA and post hoc Tukey tests of transformed values of \% Growth $[\text { month }]^{-1}(\mathrm{Ln})$ and \% Survivorship (Arcsin) binned according to approach.

Table S4. "Global" mean $( \pm \mathrm{SE})$ relative return on effort (RRE) score according to coral species across datasets.

Table S5. Summary data collected from nursery corals at from Opal Reef (as of August 2018).

Figure S1. Visualization of the dynamic nature of the RRE score is potentially as important as the score itself.

Figure S2. Photograph showing Opal Reef platforms (first site, May 2018).

Figure S3. Box plots of transformed values of \% Growth $[\text { month }]^{-1}(\mathrm{Ln})$ and $\%$ Survivorship (Arcsin) binned according to time.

Figure S4. Comparison of untransformed \% Growth $[\text { month }]^{-1}$ (Eq. 1) and \% Survivorship versus initial propagule size.

Figure S5. Box plots of transformed values of \% Growth $[\text { month }]^{-1}$ (Ln) and \% Survivorship (Arcsin) binned according to approach.

Figure S6. Box plots of relative return-on-effort (RRE) scores retrieved from plots of transformed \% Growth versus \% Survivorship (Fig. 1) binned according to year of study.

Figure S7. Comparison of untransformed \% Growth [month $]^{-1}$ (Eq. 1) versus initial propagule size.

Received: 8 October, 2018; First decision: 30 November, 2018; Revised: 14 December, 2018; Accepted: 15 December, 2018 